\title{
Main Patterns of Fetal Cardiac Remodeling
}

\author{
Fàtima Crispi $^{\mathrm{a}}$ Álvaro Sepúlveda-Martínez ${ }^{\mathrm{a}, \mathrm{b}} \quad$ Francesca Crovetto $^{\mathrm{a}}$ \\ Olga Gómez ${ }^{a}$ Bart Bijnens ${ }^{c, d}$ Eduard Gratacós ${ }^{a}$
}

${ }^{a}$ Fetal Medicine Research Center, BCNatal - Barcelona Center for Maternal-Fetal and Neonatal Medicine (Hospital Clínic and Hospital Sant Joan de Déu), Institut Clínic de Ginecologia Obstetricia i Neonatologia, Institut d'Investigacions Biomèdiques August Pi i Sunyer, Universitat de Barcelona, Centre for Biomedical Research on Rare Diseases (CIBER-ER), Barcelona, Spain; ${ }^{b}$ Fetal Medicine Unit, Department of Obstetrics and Gynecology, Hospital

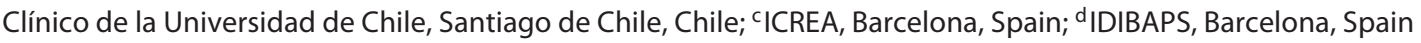

\section{Keywords}

Fetal heart · Prenatal development · Intrauterine growth restriction · Assisted reproductive technologies · Congenital heart disease $\cdot$ Echocardiography

\section{Abstract}

The heart is a central organ in the fetal adaptation to an adverse environment. Fetal cardiac changes may persist postnatally and increase the risk of cardiovascular disease in adulthood. Knowledge about fetal cardiac structural as well as functional remodeling has radically improved over the last few years. As it occurs in postnatal life, the fetal heart remodels - changing its structure and shape - to adapt to an insult. Several conditions have been reported to be associated with fetal cardiac remodeling including intrauterine growth restriction, diabetes, exposure to antiretroviral drugs, conception by assisted reproductive technologies, pulmonary stenosis, and other congenital heart diseases. Here we summarized the main observable patterns of cardiac remodeling, i.e., globular shape, hypertrophy without dilation, and hypertrophy with cardiomegaly. We discuss the potential pathophysiology behind different types of remodeling. Defining precisely the distinct patterns of fetal cardiac remodeling is critical for advancing in the understanding of fetal cardiovascular programming and its consequences on adult health, and potentially for the design of preventive strategies that might have an impact on long-term cardiovascular health.

(c) 2020 S. Karger AG, Basel

"Experentia docet"

- Tacitus (Roman)

\section{Introduction}

The heart is the first functional organ to develop. This emphasizes its critical role in distributing blood, nutrients, oxygen, and waste to and from the developing fetus. The heart is a kinetic organ whose structure and form change during development and disease [1]. The prenatal environment will drive the development of the heart so that it has an optimal structure and function given the conditions present. This way, the heart is es-

Fàtima Crispi and Álvaro Sepúlveda-Martínez contributed equally to this work. karger@karger.com

www.karger.com/fdt

Karger" (c) 2020 S. Karger AG, Base

(1020 S. Karger AG, Basel
Fàtima Crispi

BCNatal - Barcelona Center for Maternal-Fetal and Neonatal Medicine (Hospital Clínic and Hospital Sant Joan de Déu), University of Barcelona Sabino Arana street 1, ES-08028 Barcelona (Spain)

fcrispi@clinic.cat 
sential in the fetal adaptation to a pathological environment. In the initial stages of an insult, the heart usually manages to adapt, undergoing changes in shape, structure, and size (defined as cardiac remodeling) in order to maintain an optimal and efficient function [2]. In postnatal (especially adult) life, cardiac remodeling may occur following different main patterns, which are well established [3]. Evidence on cardiac remodeling during fetal life is still recent, but available data suggest that the fetus develops distinct patterns of remodeling under different insults. In this article, we will review the main patterns of cardiac remodeling that have so far been described in utero in several physiological and pathological conditions, as well as its relationship with cardiac dysfunction and failure.

\section{Main Text}

\section{Normal Fetal Cardiac Structure and Function}

The primary function of the heart is to circulate blood in order to provide adequate perfusion of organs [4]. The heart achieves this function by first filling the ventricles (diastole) and then contracting its muscular walls around a closed chamber to generate sufficient pressure to eject blood from the ventricle into the aorta/pulmonary artery (systole) $[4,5]$. The growth of the heart during fetal development is therefore determined by the conditions it has to fulfill: blood flow and mechanical forces by and on the muscle. Essentially, heart shape and function are mainly determined by intrinsic tissue properties such as myocyte contractility, fiber orientation, tissue elasticity, heart geometry, as well as boundary conditions such as segment interaction, loading conditions, electrical activation, and myocardial perfusion. Additionally, intrinsic myocardial maturation as well as the fetal peripheral blood circulation and demand are also critical factors.

\section{The Heart as a Central Organ in the Fetal Adaptive} Response to Insults

The heart is a key organ in the fetal adaptive response to insults such as toxins, hypoxia, undernutrition, or pressure/volume overload. The fetal heart will adapt by changing its structure and shape in order to ensure blood supply to organs in an optimal way, where myocytes operate in their most efficient (mechanical) conditions; this process is defined as cardiac remodeling. The pattern of cardiac remodeling depends on the type of insult and time and duration of exposure. Distinctive patterns of re- modeling are shape change (more globular ventricles, optimizing myocyte forces in response to pressure overload), myocardial hypertrophy (improving contractility as response to pressure overload), or cavity dilation (increasing ejection potential, usually secondary to volume overload) or hypoplasia.

Changes in cardiac structure and shape are usually accompanied by subclinical changes in the heart's functioning; this remodeling process can lead to cardiac dysfunction. Fetal cardiac dysfunction may affect the systolic the ability to pump enough blood into circulation - and/ or diastolic - the ability to relax the myocardium to fill the ventricles - function. Changes in cardiac architecture may also lead to alterations in ventricular rotation and torsion [6-8]. In most clinical situations, fetal cardiac dysfunction is subclinical. However, if the insult is severe enough or persists, impaired cardiac pumping to maintain systemic and/or pulmonary circulations and impaired myocardial relaxation with increased ventricular filling pressure lead to clinical cardiac failure, which in utero essentially manifests as fetal hydrops.

\section{Main Determinants of Fetal Cardiac Remodeling}

The heart adaptation to noxas results in increasing myocardial mass and/or dilatation. Cardiac remodeling can affect the whole heart (i.e., cardiomegaly) or preferably affect the right/left ventricles/atria. In the fetus, changes are typically more prominent in the right side, most likely reflecting the dominance of the right side of the heart prenatally and a higher susceptibility to pressure overload of the right compared with the left ventricle. Actually, the right ventricle acts as the "systemic" ventricle in fetal life being responsible to perfuse the fetal organs and also the placenta.

An abnormal environment can affect the fetal heart through different mechanisms:

Direct Myocardial Damage. Myocardium could directly be affected by a toxin (i.e., antiretroviral drugs) [9], hypoxia/ischemia (i.e., placental insufficiency [10] or ane$\mathrm{mia}$ ), or genetic cardiomyopathies inducing global/regional edema, inflammation, fiber disarray, fibrosis, or cell loss. Cardiac toxicity is frequently compensated by myocardial hypertrophy, compensating for cell loss or decreased intrinsic contractility.

Pressure Overload. Pulmonary/aortic stenosis [5, 11], twin-to-twin transfusion syndrome [12-14], placental disease [15], and conception by assisted reproductive technologies [16] induce pressure overload and trigger a fetal heart response by initially changing to a more globular shape (to better tolerate wall stress) and subsequently further increas- 


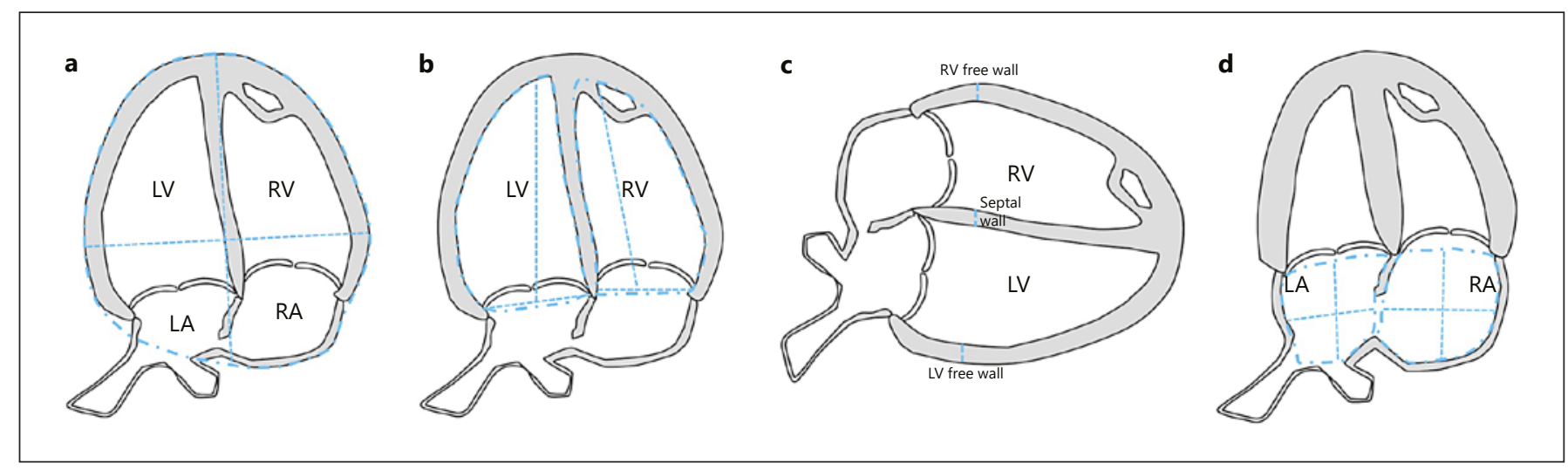

Fig. 1. Fetal cardiac schemes illustrating the measurement of cardiac diameters and area (a), ventricular diameters and areas (b) and myocardial wall thicknesses at end-diastole (c), and atrial diameters and areas at end-systole (d). LV, left ventricle; RV, right ventricle; LA, left atria; RA, right atria.

ing the contractile force (myocardial hypertrophy) as well as decreasing the inner diameter of the chamber.

Volume Overload. Prenatal volume overload occurs in fetal anemia [17], vascularized tumors [17], severe intrauterine growth restriction $[6,15,18,19]$, abnormal venous drainage [20], or twin-to-twin transfusion syndrome $[12,14]$. The natural response is to enlarge heart dimensions (cardiac dilation), thus increasing the stroke volume with every beat, in order to better manage the increased blood volume.

Thus, the fetal heart usually adapts to noxas by changing shape, increasing myocardial mass, and/or dilating. While cardiac remodeling is thus induced to compensate adverse conditions, there are some major differences between fetal and adult remodeling. Given that fetal remodeling takes place during development, the intrinsic shape and (micro-)structure can still change, while in adult life these changes are much more limited. Additionally, several fetal insults disappear with birth, so that a heart optimized for specific fetal conditions might not be ideal for postnatal life.

Patterns of ventricular remodeling are usually defined according to the ventricular size and shape, the presence of myocardial hypertrophy, and the relation between myocardial mass and cavity dimensions. Concentric remodeling is defined by increased mass-to-cavity ratio and is usually observed when a pressure leads to cardiomyocyte hypertrophy or hyperplasia. On the contrary, massto-cavity ratio is preserved in eccentric hypertrophy and is typically secondary to volume loading inducing myocyte lengthening. Atrial remodeling is less common and usually occurs in the form of dilation.

Fetal Cardiac Remodeling
Echocardiographic Evaluation of Fetal Cardiac Shape and Size

In adulthood, the presence and pattern of cardiac remodeling is assessed by imaging to estimate ventricular and atria volumes, left ventricular mass (to define myocardial hypertrophy), and relative wall thickness or massto-cavity ratio. Echocardiography is the most used modality, but cardiac magnetic resonance or computed tomography can provide more accurate measurements. While radiation excludes the use of computed tomography for fetal assessment, the relatively limited use in utero, the relatively small fetal heart size, the elevated fetal heart rate, and the lack of fetal ECG also hamper the use of cardiac magnetic resonance in prenatal life. Therefore, fetal echocardiography is considered the best available option for assessing fetal cardiac remodeling by measuring heart, ventricular, and atria dimensions (diameters and areas) and myocardial wall thickness.

Fetal 2D echocardiography has demonstrated to be highly feasible and reproducible for assessing fetal cardiac structure and shape when a standardized and strict methodology is followed (Fig. 1) [21, 22]. An apical or basal four-chamber view permits the measurement of cardiac, ventricular, and atrial dimensions (diameters and areas) and shape (sphericity by dividing longitudinal per transverse diameters) using specific landmarks. A lateral 2D or M-mode four-chamber view is recommended for myocardial wall thickness measurement. Most cardiac and ventricular parameters are standardized to be measured at end-diastole (at maximal ventricular distension at the frame when the atrioventricular valves close), while atrial dimensions are usually recommended to be as- 
Table 1. Fetal echocardiographic parameters proposed to evaluate patterns of cardiac remodeling in prenatal life

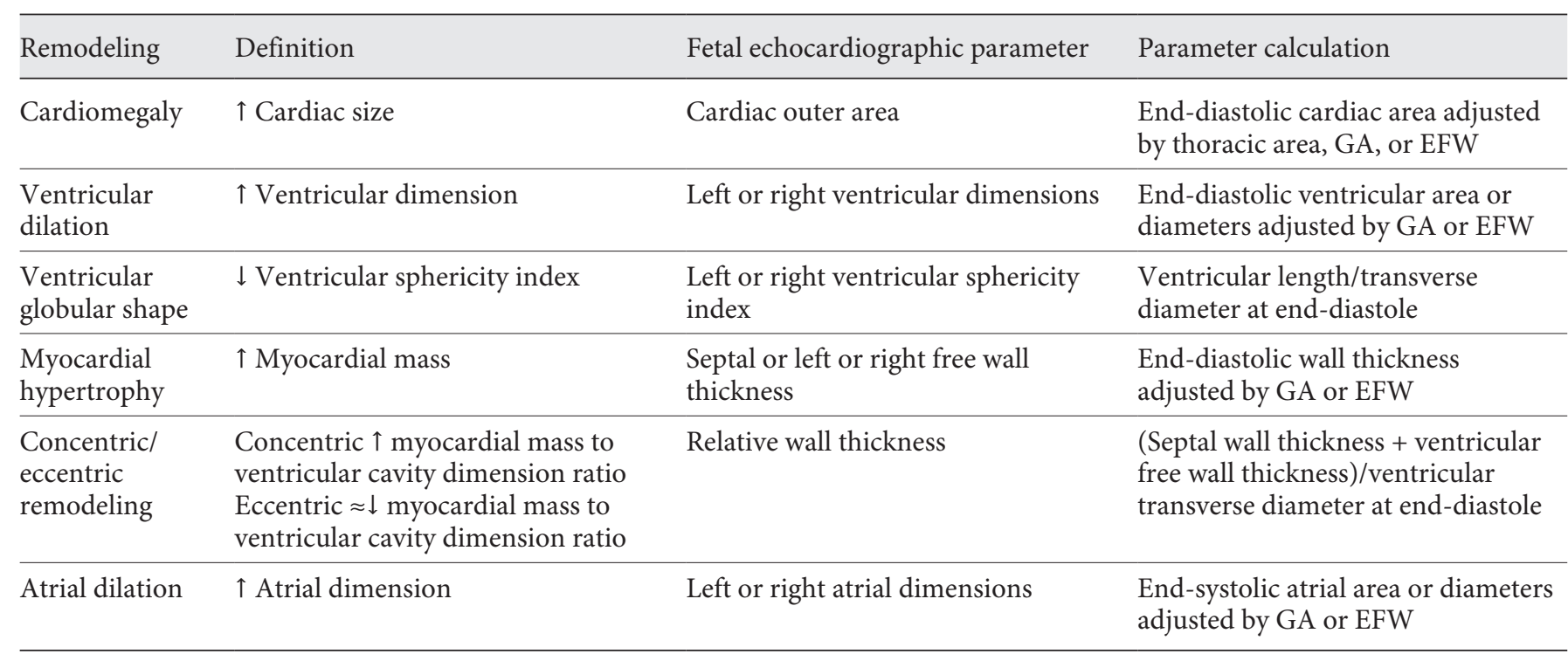

GA, gestational age; EFW, estimated fetal weight.

sessed at end-systole (maximal atrial distension just before the opening of atrioventricular valves). Standard criteria and nomograms for most fetal cardiac dimensions throughout gestation are available using standard 2D echo $[21,22]$ and also a novel application of speckle tracking echocardiography [23]. Despite no actual consensus, patterns of cardiac remodeling in fetal life are usually described reporting cardiac, ventricular, and atrial areas, ventricular sphericity indices, septal wall thickness, and relative wall thickness adjusted by gestational age or estimated fetal weight (Table 1).

\section{Patterns of Fetal Cardiac Remodeling}

We here summarize the main features of the most commonly described patterns of fetal cardiac remodeling (Fig. 2).

\section{Globular Shape}

A globular heart is characterized by non-hypertrophic, more spherical ventricles with normal cardiac size (Fig. 2a) usually associated with a mild decrease in longitudinal motion and impaired relaxation $[6,15]$. This pattern has been described in mild cases of fetal growth restriction diagnosed late in pregnancy where presumably a mild degree of placental insufficiency increases pressure and induces a compensatory change in the heart shape without the need of myocardial hypertrophy. The proposed mechanism is that the fetal heart must pump against a more resistant placenta (pressure overload) and remodels to a more globular shape in order to reduce the ventricular radius of curvature and better tolerate the increased wall stress.

Myocardial Hypertrophy with Preserved Cardiac Size

Persistent pressure overload induces globular shape but also increased myocardial wall thickness and reduced ventricular cavities (increased relative wall thickness) maintaining a normal outer heart size. This pattern has been described in cases of severe pulmonary stenosis characterized by thicker septal and right myocardial walls together with smaller right ventricular cavity and signs of diastolic dysfunction [11]. The chronic right pressure overload induces right ventricular hypertrophy as well as a more spherical configuration with a smaller inner diameter in order to generate higher force and pressure with lower stress on the individual myocytes and less energy consumption.

Fetuses conceived by assisted reproductive technologies also display a cardiac phenotype of concentric ventricular remodeling together with atrial dilatation, decreased longitudinal motion, and impaired relaxation [16, 24]. It is postulated to be a response to a predominantly right pressure overload, and it is consistent with studies reporting increased pulmonary pressures in children conceived by assisted reproductive technologies. The observed dilated atria have been suggested to be explained by a ventricular early filling problem that needs to be com- 


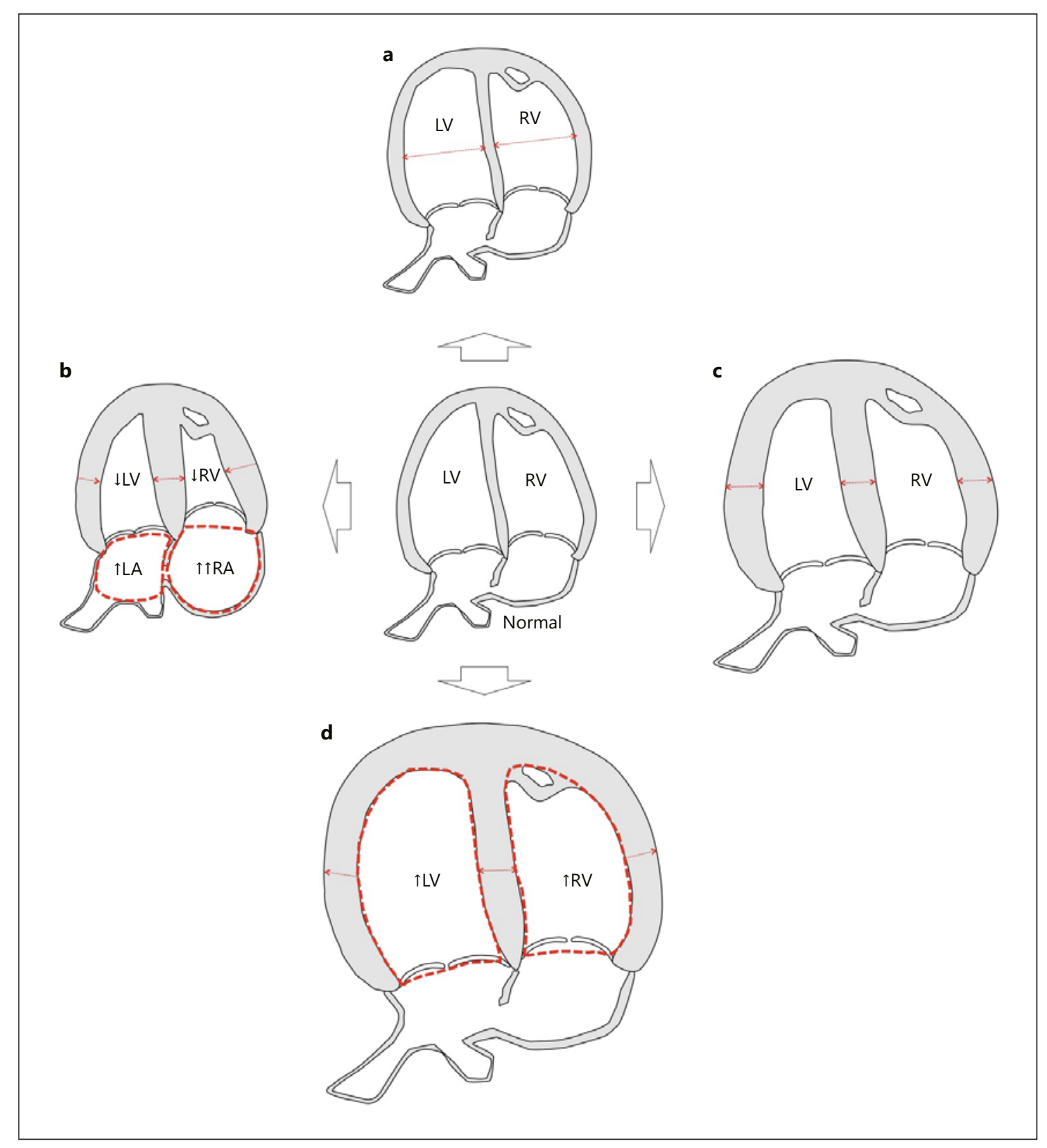

Fig. 2. Fetal cardiac schemes illustrating patterns of fetal cardiac remodeling. a Globular shape in late mild fetal growth restriction. b Myocardial hypertrophy and atrial dilation in fetuses conceived by assisted reproductive technologies. c Myocardial hypertrophy and cardiomegaly in fetuses exposed to maternal HIV infection and antiretroviral drugs. d Myocardial hypertrophy, ventricular dilation, and cardiomegaly in early severe fetal growth restriction. $\mathrm{LV}$, left ventricle; $\mathrm{RV}$, right ventricle; $\mathrm{LA}$, left atria; $\mathrm{RA}$, right atria.

pensated by a larger atrial contribution; increased pressure also results in dilated atria. A pattern of myocardial hypertrophy with reduced ventricular cavities has also been described in the early postnatal life of preterm babies [23], most likely related to relative pressure overload during the first days of life of preterm newborns.

\section{Myocardial Hypertrophy with Cardiomegaly}

The first reports of fetal myocardial hypertrophy with cardiomegaly were associated with maternal diabetes and proposed to be mediated by fetal hyperinsulinemia [24]. It is characterized by enlarged hearts, thickening of the myocardium, and increased shortening fraction and lon- 
gitudinal motion [25]. Myocardial hypertrophy particularly affects the septum and may lead to left ventricular outflow obstruction [24].

The use of antiretroviral drugs - particularly zidovudine, which induces cardiac mitochondrial toxicity and thus decreased intrinsic contractility - during pregnancy has been reported to be associated with a characteristic pattern of concentric hypertrophy with larger hearts, increased myocardial wall thickness, and more spherical ventricles (Fig. 2c). In addition, 35\% of the cases present mild pericardial effusion and most of them show signs of systolic and diastolic dysfunction (reduced longitudinal motion and impaired relaxation) $[8,26]$. It has been postulated to be a compensatory fetal cardiac hypertrophic response to myocardial damage associated with toxicity.

Cases of early-onset fetal growth restriction with severe placental insufficiency are characterized by cardiomegaly, myocardial hypertrophy, and globular dilated ventricles [15] (Fig. 2d). This eccentric hypertrophy is usually associated with mild pericardial effusion, decreased longitudinal function, impaired relaxation [14, 27], and abnormal regional deformation [28], with preserved ejection fraction in most cases. The pathophysiology of cardiac remodeling and dysfunction under placental insufficiency has been proposed to be caused by complex interactions [15]. First, the reduction in oxygen and nutrients supply would increase volume load and also directly disrupt normal cardiomyocyte growth and fiber architecture, and therefore, decrease its contractility. Secondly, placental resistance leads to chronic pressure overload. The fetal heart would react to pressure overload by shifting to a more spherical shape and thickening myocardial walls (hypertrophy). In addition, volume overload would be responsible for cardiomegaly and mild pericardial effusion.

Apart from the above mentioned, other patterns of fetal cardiac remodeling have also been reported describing ventricular elongation or hypoplasia, thinning of the ventricular walls, asymmetric remodeling, endocardial fibroelastosis, etc. [25-32].

\section{Conclusion}

In this review we summarized the main patterns of cardiac remodeling described in fetal life. The description of patterns provided here may help in the evaluation of cardiac remodeling. Such assessment is now easier since nomograms for most cardiac dimensions have recently been published $[21,22,33]$. A precise description of the morphological changes in the fetal heart may help comparison among research studies and contributes to understanding the pathophysiology of cardiovascular changes occurring under various pregnancy conditions (i.e., placental insufficiency, exposure to toxics, assisted reproductive technologies, valve stenosis). Likewise, identifying fetal cardiac remodeling might be an opportunity to improve postnatal cardiovascular health.

Cardiac remodeling can occur at any stage of life. However, while postnatal cardiac remodeling reverts after treating the cause, cardiac remodeling occurring in utero might persist postnatally even after the trigger has disappeared. Actually, postnatal persistence of fetal cardiac remodeling has been demonstrated in several conditions such as fetal growth restriction, maternal diabetes, exposure to antiretroviral drugs, prematurity, or assisted reproductive technologies $[9,10,16,34-38]$. This phenomenon is explained by the fetal programming hypothesis that proposes a prenatal origin for some adult cardiovascular diseases. The fetal programming hypothesis postulates that insults in utero occur in a critical stage of development and structural and functional changes in key organs during this stage of life will persist postnatally explaining a greater susceptibility to disease in adulthood. This notion is strongly supported by large epidemiological and experimental animal studies demonstrating the relationship between low birth weight with cardiovascular disease and mortality in adulthood [30]. The rapid cell proliferation and differentiation during fetal growth are very sensitive to any change in environment and will lead to permanent alterations in the structural and functional constitution. Actually, fetal life is a critical period of development when organs are being programmed to adapt to the environment. Any insult occurring during this critical period can result in developmental adaptations that produce permanent structural, physiological, and metabolic changes, thereby predisposing an individual to disease in adult life.

Understanding fetal adaptation to the in utero environment by assessing fetal cardiac remodeling might be useful to monitor the fetus but also to identify those cases with increased risk of cardiovascular disease later in life. This opens an opportunity for early selection of individuals that might benefit from preventive measures to improve cardiovascular health. For example, it has been demonstrated that breastfeeding or a healthy diet in infancy could ameliorate the cardiovascular remodeling associated to fetal growth restriction [15] or prematurity [31]. Fetal life seems to constitute a unique window of opportunity for the early diagnosis and prevention of cardiovascular disease. 


\section{Statement of Ethics}

The authors have no ethical conflicts to disclose.

\section{Disclosure Statement}

The authors have no conflicts of interest to declare.

\section{Funding Sources}

This project has been partially funded with support of the Erasmus+ Programme of the European Union (Framework Agreement number: 2013-0040). This publication reflects the views only of the authors, and the Commission cannot be held responsible for any use which may be made of the information contained therein. Additionally, the research leading to these results has received funding form "la Caixa" Foundation under grant agreement LCF/ PR/GN14/10270005, the Instituto de Salud Carlos III (PI15/00130, PI15/00263, PI17/00675, PI18/00073) integrados en el Plan Nacional de I+D+I y cofinanciados por el ISCIII-Subdirección General de Evaluación y el Fondo Europeo de Desarrollo Regional (FEDER) "Una manera de hacer Europa," the Centro de Investigación
Biomédica en Red de Enfermedades Raras (ERPR04G719/2016), Cerebra Foundation for the Brain Injured Child (Carmarthen, Wales, UK), and AGAUR 2017 SGR grant No. 1531.

\section{Author Contributions}

Fàtima Crispi has participated in the conception and design of the review, drafted the work, approved the final version, and agreed on the accuracy and integrity of the work. Álvaro Sepúlveda-Martínez has participated in the conception and design of the review, drafted the work, approved the final version, and agreed on the accuracy and integrity of the work. Francesca Crovetto has participated in the design of the review, drafted the work, approved the final version, and agreed on the accuracy and integrity of the work. Olga Gómez has participated in the design of the review, revised it critically for important intellectual content, approved the final version, and agreed on the accuracy and integrity of the work. Bart Bijnens has participated in the design of the review, revised it critically for important intellectual content, approved the final version, and agreed on the accuracy and integrity of the work. Eduard Gratacós has participated in the design of the review, revised it critically for important intellectual content, approved the final version, and agreed on the accuracy and integrity of the work.

\section{References}

1 Andrés-Delgado L, Mercader N. Interplay between cardiac function and heart development. Biochim Biophys Acta. 2016 Jul;1863(7 7 Pt B):1707-16.

2 Opie LH, Commerford PJ, Gersh BJ, Pfeffer MA. Controversies in ventricular remodelling. Lancet. 2006 Jan;367(9507):356-67.

3 Rodriguez CJ, Diez-Roux AV, Moran A, Jin Z, Kronmal RA, Lima J, et al. Left ventricular mass and ventricular remodeling among Hispanic subgroups compared with non-Hispanic blacks and whites: MESA (Multi-Ethnic Study of Atherosclerosis). J Am Coll Cardiol. 2010 Jan;55(3):234-42.

4 Mayet J, Hughes A. Cardiac and vascular pathophysiology in hypertension. Heart. 2003 Sep;89(9):1104-9.

5 Bijnens B, Cikes M, Butakoff C, Sitges M, Crispi F. Myocardial motion and deformation: what does it tell us and how does it relate to function? Fetal Diagn Ther. 2012;32(1-2) 5-16.

6 Pérez-Cruz M, Cruz-Lemini M, Fernández MT, Parra JA, Bartrons J, Gómez-Roig MD, et al. Fetal cardiac function in late-onset intrauterine growth restriction vs small-for-gestational age, as defined by estimated fetal weight, cerebroplacental ratio and uterine artery Doppler. Ultrasound Obstet Gynecol. 2015 Oct;46(4):465-71.

7 Thakur V, Fouron JC, Mertens L, Jaeggi ET. Diagnosis and management of fetal heart failure. Can J Cardiol. 2013 Jul;29(7):759-67.
8 Patey O, Carvalho JS, Thilaganathan B. Left ventricular torsional mechanics in term fetuses and neonates. Ultrasound Obstet Gynecol. 2020 Feb;55(2):233-41.

9 García-Otero L, López M, Gómez O, Goncé A, Bennasar M, Martínez JM, et al. Zidovudine treatment in HIV-infected pregnant women is associated with fetal cardiac remodelling. AIDS. 2016 Jun;30(9):1393-401.

10 Crispi F, Bijnens B, Figueras F, Bartrons J, Eixarch E, Le Noble F, et al. Fetal growth restriction results in remodeled and less efficient hearts in children [Internet]. Circulation. 2010 Jun;121(22):2427-36. Available from: http://circ.ahajournals.org/cgi/doi/ 10.1161/CIRCULATIONAHA.110.937995

11 Guirado L, Crispi F, Masoller N, Bennasar M, Marimon E, Carretero J, et al. Biventricular impact of mild to moderate fetal pulmonary valve stenosis. Ultrasound Obstet Gynecol. 2018 Mar;51(3):349-56.

12 Ortiz JU, Masoller N, Gómez O, Bennasar M, Eixarch E, Lobmaier SM, et al. Rate and Outcomes of Pulmonary Stenosis and Functional Pulmonary Atresia in Recipient Twins with Twin-Twin Transfusion Syndrome. Fetal Diagn Ther. 2017;41(3):191-6

13 Eschbach SJ, Boons LS, Van Zwet E, Middeldorp JM, Klumper FJ, Lopriore E, et al. Right ventricular outflow tract obstruction in complicated monochorionic twin pregnancy. Ultrasound Obstet Gynecol. 2017 Jun;49(6): $737-43$.
14 Eschbach SJ, Ten Harkel AD, Middeldorp JM, Klumper FJ, Oepkes D, Lopriore E, et al. Acquired right ventricular outflow tract obstruction in twin-to-twin transfusion syndrome; a prospective longitudinal study. Prenat Diagn. 2018 Dec;38(13):1013-9.

15 Rodríguez-López M, Cruz-Lemini M, Valenzuela-Alcaraz B, Garcia-Otero L, Sitges M, Bijnens B, et al. Descriptive analysis of different phenotypes of cardiac remodeling in fetal growth restriction. Ultrasound Obstet Gynecol. 2017 Aug;50(2):207-14.

16 Valenzuela-Alcaraz B, Crispi F, Bijnens B, Cruz-Lemini M, Creus M, Sitges M, et al. Assisted reproductive technologies are associated with cardiovascular remodeling in utero that persists postnatally. Circulation. 2013 Sep;128(13): 1442-50.

17 Davey B, Szwast A, Rychik J. Diagnosis and management of heart failure in the fetus. Minerva Pediatr. 2012 Oct;64(5):471-92.

18 Garcia-Canadilla P, Rudenick PA, Crispi F, Cruz-Lemini M, Palau G, Camara O, et al. A computational model of the fetal circulation to quantify blood redistribution in intrauterine growth restriction. PLOS Comput Biol. 2014 Jun;10(6):e1003667.

19 Crispi F, Figueras F, Cruz-Lemini M, Bartrons J, Bijnens B, Gratacos E. Cardiovascular programming in children born small for gestational age and relationship with prenatal signs of severity [Internet]. Am J Obstet Gynecol. 2012 Aug;207(2):121.e1-9. 
20 Paladini D, Pistorio A, Wu LH, Meccariello G, Lei T, Tuo G, et al. Prenatal diagnosis of total and partial anomalous pulmonary venous connection: multicenter cohort study and meta-analysis. Ultrasound Obstet Gynecol. 2018 Jul;52(1):24-34.

21 Sepúlveda-Martínez A, García-Otero L, Soveral I, Guirado L, Valenzuela-Alcaraz B, Torres X, et al. Comparison of 2D versus Mmode echocardiography for assessing fetal myocardial wall thickness. J Matern Fetal Neonatal Med. 2019 Jul;32(14):2319-27.

22 García-Otero L, Gómez O, Rodriguez-López M, Torres X, Soveral I, Sepúlveda-Martínez Á, et al. Nomograms of Fetal Cardiac Dimensions at 18-41 Weeks of Gestation. Fetal Diagn Ther. 2019 Jan;1-12.

23 DeVore GR, Klas B, Satou G, Sklansky M. 24-segment sphericity index: a new technique to evaluate fetal cardiac diastolic shape. Ultrasound Obstet Gynecol. 2018 May;51(5):6508.

24 Valenzuela-Alcaraz B, Serafini A, SepulvedaMartínez A, Casals G, Rodríguez-López M, Garcia-Otero L, et al. Postnatal persistence of fetal cardiovascular remodelling associated with assisted reproductive technologies: a cohort study. BJOG. 2019 Jan;126(2):291-8.

25 Cruz-Lemini M, Valenzuela-Alcaraz B, Granados-Montiel J, Martínez JM, Crispi F, Gratacós E, et al. Characterizing cardiac dysfunction in fetuses with left congenital diaphragmatic hernia. Prenat Diagn. 2018 May; 38(6):422-7.
26 Patey O, Carvalho JS, Thilaganathan B. Perinatal changes in cardiac geometry and function in growth-restricted fetuses at term. Ultrasound Obstet Gynecol. 2019 May;53(5): 655-62.

27 DeVore GR, Jone PN, Satou G, Sklansky M, Cuneo BF. Aortic Coarctation: A Comprehensive Analysis of Shape, Size, and Contractility of the Fetal Heart. Fetal Diagn Ther. 2019 May 27:1-11.

28 Gottschalk I, Strizek B, Menzel T, Herberg U, Breuer J, Brockmeier K, et al. Severe Pulmonary Stenosis or Atresia with Intact Ventricular Septum in the Fetus: The Natural History. Fetal Diagn Ther. 2019 Aug 27:1-9.

29 Brooks PA, Khoo NS, Hornberger LK. Systolic and diastolic function of the fetal single left ventricle. J Am Soc Echocardiogr. 2014 Sep; 27(9):972-7.

30 Brooks PA, Khoo NS, Mackie AS, Hornberger LK. Right ventricular function in fetal hypoplastic left heart syndrome. J Am Soc Echocardiogr. 2012 Oct;25(10):1068-74.

31 Mallmann MR, Herberg U, Gottschalk I, Strizek B, Hellmund A, Geipel A, et al. Fetal Cardiac Intervention in Critical Aortic Stenosis with Severe Mitral Regurgitation, Severe Left Atrial Enlargement, and Restrictive Foramen Ovale. Fetal Diagn Ther. 2019 Oct 8:1-8.

32 Gardiner HM, Kovacevic A, Tulzer G, Sarkola T, Herberg U, Dangel J, et al.; Fetal Working Group of the AEPC. Natural history of 107 cases of fetal aortic stenosis from a European multicenter retrospective study. Ultrasound Obstet Gynecol. 2016 Sep;48(3):373-81.
33 DeVore GR, Cuneo B, Klas B, Satou G, Sklansky M. Comprehensive Evaluation of Fetal Cardiac Ventricular Widths and Ratios Using a 24-Segment Speckle Tracking Technique. J Ultrasound Med. 2019 Apr;38(4):1039-47.

34 Patey O, Carvalho JS, Thilaganathan B. Perinatal changes in fetal cardiac geometry and function in diabetic pregnancy at term. Ultrasound Obstet Gynecol. 2019 Nov;54(5):63442.

35 Maroules CD, Khera A, Ayers C, Goel A, Peshock RM, Abbara S, et al. Cardiovascular outcome associations among cardiovascular magnetic resonance measures of arterial stiffness: the Dallas heart study [Internet]. J Cardiovasc Magn Reson. 2014 May;16(1):33. Available from: http://www.pubmedcentral. nih.gov/articlerender.fcgi?artid $=4031496 \&$ to $\mathrm{ol}=$ pmcentrez\&rendertype $=$ abstract

36 Gandhi JA, Zhang XY, Maidman JE. Fetal cardiac hypertrophy and cardiac function in diabetic pregnancies. Am J Obstet Gynecol. 1995 Oct;173(4):1132-6.

37 Sarvari SI, Rodriguez-Lopez M, Nuñez-Garcia M, Sitges M, Sepulveda-Martinez A, Camara O, et al. Persistence of Cardiac Remodeling in Preadolescents with Fetal Growth Restriction. Circ Cardiovasc Imaging. 2017 Jan; 10(1): 005270

38 Gardiner HM, Pasquini L, Wolfenden J, Kulinskaya E, Li W, Henein M. Increased periconceptual maternal glycated haemoglobin in diabetic mothers reduces fetal long axis cardiac function. Heart. 2006 Aug;92(8):112530 . 